\title{
ZERO DIVISORS IN NOETHERIAN-LIKE RINGS
}

\author{
BY \\ E. GRAHAM EVANS, JR. $\left({ }^{1}\right),\left({ }^{2}\right)$
}

\begin{abstract}
The zero divisors of $R / I$ for every ideal $I$ of a Noetherian ring is a finite union of primes. We take this property as a definition and study the class of rings so defined. Such rings are stable under localization and quotients. They are not stable under integral closure and are highly unstable under polynomial adjunction. The length of maximal $R$ sequences is well defined on them.

In this paper all rings are commutative with unit and all modules are unitary.
\end{abstract}

Definitions. Let $R$ be a ring and $M$ a nonzero $R$ module. Then the zero divisors on $M, Z_{R}(M)$, is $\{r \in R \mid \exists 0 \neq m \in M$ with $r m=0\}$. We will drop the $R$ when there is no possibility of confusion. $R$ is a zero divisor ring, Z.D. ring, if $Z_{R}(R / I)$ is a finite union of prime ideals for all ideals $I$ of $R$. $M$ is a zero divisor module, Z.D. module, if $Z_{R}\left(M / M^{\prime}\right)$ is a finite union of prime ideals for all submodules $M^{\prime}$ of $M$.

LEMMA 1. Let $R$ be a ring, $M$ an $R$ module, and $S$ a multiplicatively closed subset of $R$ contained in $R-Z(M)$. Then

$$
Z_{R}\left(M_{S}\right)=Z_{R}(M)
$$

and

$$
Z_{R_{S}}\left(M_{S}\right)=Z_{R}(M) \cdot R_{S}
$$

Proof. (1) If $r \in Z_{R}(M)$, then there exists $0 \neq m \in M$ with $r m=0$. Then $r m / 1=0$ in $R_{S}$. But $m / 1 \neq 0 / 1$ in $M_{S}$ since $S \subset R-Z_{R}(M)$. Thus $r \in Z_{R}\left(M_{S}\right)$.

If $r \in Z_{R}\left(M_{S}\right)$, then there exists $0 / 1 \neq m / s$ with $\mathrm{rm} / \mathrm{s}=0$. Then $\mathrm{rsm} / \mathrm{s}=\mathrm{rm} / 1=0$. Hence, there is a $t \in S$ such that $t r m=0$. But $t m \neq 0$ and $r t m=0$ implies $r \in Z_{R}(M)$.

(2) is immediate from (1).

LEMmA 2. Let $M$ be an $R$ module and $S \subset R-Z(M)$. If $Z_{R_{S}}\left(M_{S}\right)=P_{1} \cup \cdots \cup P_{n}$ where $P_{i}=p_{i_{S}}$ with $P_{i}$ and $p_{i}$ prime ideals, then $Z_{R}(M)=p_{1} \cup \cdots \cup p_{r}$.

Proof. Let $r \in p_{1} \cup \cdots \cup p_{r}$ then pick a nonzero $m / 1 \in M_{S}$ with $m \in M$ such that $(r / 1)(m / 1)=0 / 1$. Then there is a $t \in S$ with $t r m=0$. But $t m \neq 0$. Hence $r \in Z_{R}(M)$.

Received by the editors July 10, 1970.

AMS 1969 subject classifications. Primary 1325; Secondary 1340, 1310.

Key words and phrases. Noetherian rings, zero divisors, integral closure, polynomials, $R$ sequences, Serre subcategory, Laskerian, primary ideals, valuation rings.

( ${ }^{1}$ This paper is part of the author's doctoral dissertation written while he was a NASA trainee at the University of Chicago.

$\left({ }^{2}\right)$ Current address: Massachusetts Institute of Technology, Cambridge, Massachusetts 02139.

Copyright (C) 1971, American Mathematical Society 
Let $a \in Z_{R}(M)$. Then $a / 1 \in Z_{R_{S}}\left(M_{S}\right)$ by Lemma 1 . Say $a / 1=p / s$ with $p \in p_{1}$ and $s \in S$. Then there exists a $t \in S$ with $t s a=t p$. Hence $t s a \in p_{1}$ but $t s \notin p_{1}$ since $p_{1} \subset Z_{R}(M)$. Thus $a \in p_{1}$ as desired.

Proposition 3. (1) If $R$ is a Z.D. ring, then $R_{S}$ is a Z.D. ring for all multiplicatively closed subsets $S$ of $R$.

(2) If $M$ is a Z.D. R module, then $M_{S}$ is a Z.D. $R_{S}$ module for all multiplicatively closed sets $S$ of $R$.

Proof. (1) Let $I^{\prime}$ be an ideal of $R_{S}$ and $I$ be the inverse image of $I^{\prime}$ under the map $f: R \rightarrow R_{S}$ given by $f(r)=r / 1$. Then $S \subset R-Z(R / I)$. For, if $s r \in I$, then $s r / 1 \in I^{\prime}$. Hence $(1 / s)(s r / 1)=r / 1 \in I^{\prime}$ and $r \in I . Z(R / I)$ is a finite union of primes by hypothesis. Hence, $Z_{R_{S}}\left(R_{S} / I^{\prime}\right)$ is a finite union of primes by Lemma 1(2).

(2) Let $M^{\prime}$ be an $R_{S}$ submodule of $M_{S}$ and $M^{\prime \prime}$ be the inverse image of $M^{\prime}$ in $M$. Then $S \subset R-Z_{R}\left(M / M^{\prime \prime}\right), Z_{R}\left(M_{S} / M^{\prime}\right)=Z_{R}\left(M / M^{\prime \prime}\right)$, and $Z_{R_{S}}\left(M_{S} / M^{\prime}\right)=Z_{R}\left(M / M^{\prime \prime}\right) R_{S}$. Hence, $M_{S}$ is a Z.D. $R_{S}$ module.

Proposition 4. Let $R$ be any ring. Then the Z.D. $R$ modules form a Serre subcategory of all $R$ modules. That is, if $0 \rightarrow M^{\prime} \rightarrow M \rightarrow M^{\prime \prime} \rightarrow 0$ is an exact sequence of $R$ modules, then $M$ is a Z.D. $R$ module if and only if $M^{\prime}$ and $M^{\prime \prime}$ are.

Proof. Let $M^{\prime}$ and $M^{\prime \prime}$ be Z.D. modules and $N$ a submodule of $M$. Then the sequence

$$
0 \rightarrow M^{\prime} / M^{\prime} \cap N \rightarrow M / N \rightarrow M^{\prime \prime} / M^{\prime}+N \rightarrow 0
$$

induced from the given sequence is exact. Let $S=R-Z(M / N)$. Then the tensored sequence

$$
0 \rightarrow\left(M^{\prime} / M^{\prime} \cap N\right)_{S} \rightarrow(M / N)_{S} \rightarrow\left(M^{\prime \prime} / M^{\prime}+N\right)_{S} \rightarrow 0
$$

is exact. If $Z_{R_{S}}\left((M / N)_{S}\right)$ is a finite union of primes, then $Z_{R}(M / N)$ is by Lemma 2 . $\left(M^{\prime} / M^{\prime} \cap N\right)_{S}$ and $\left(M^{\prime \prime} / M^{\prime \prime}+N\right)_{S}$ are Z.D. $R_{S}$ modules by Proposition 3 . $Z_{R_{S}}\left((M / N)_{S}\right) \subset Z_{R_{S}}\left((M / N)_{S}\right) \cup Z_{R_{S}}\left(\left(M^{\prime \prime} / M^{\prime}+N\right)_{S}\right)$ since the sequence (**) is exact. But $Z_{R_{S}}\left((M / N)_{S}\right)$ is all the nonunits of $R_{S}$. Hence

$$
Z_{R_{S}}\left((M / N)_{S}\right)=Z_{R_{S}}\left(\left(M^{\prime} / M^{\prime} \cap N\right)_{S}\right) \cup Z_{R_{S}}\left(\left(M^{\prime \prime} / M^{\prime}+N\right)_{S}\right) ;
$$

$Z_{R_{S}}\left((M / N)_{S}\right)$ is a finite union of primes; and $M$ is a Z.D. module.

If $M$ is a Z.D. module, then $M^{\prime \prime}$ is since any image of $M^{\prime \prime}$ is an image of $M$. It remains to show that submodules of Z.D. modules are Z.D. Let $M^{\prime}$ be a submodule of the Z.D. module $M$ and $M^{\prime \prime}$ a submodule of $M^{\prime}$. Then $M / M^{\prime \prime}$ is Z.D. Hence it is enough to show that submodules of Z.D. are Z.D. Hence it is enough to show $Z\left(M^{\prime}\right)$ is a finite union of primes. Let $S=R-Z\left(M^{\prime}\right)$. Then $M_{S}$ is a Z.D. $R_{S}$ module and $Z_{R_{S}}\left(M_{S}^{\prime}\right)=Z_{R}\left(M^{\prime}\right) R_{S} . Z_{R_{S}}\left(M_{S}^{\prime}\right) \subset Z_{R_{S}}\left(M_{S}\right)$ since $M_{S}^{\prime}$ is a submodule of $M_{S}$. But $Z_{R_{S}}\left(M_{S}^{\prime}\right)$ is all the nonunits of $R_{S}$ by construction. Hence $Z_{R_{S}}\left(M_{S}^{\prime}\right)=Z_{R_{S}}\left(M_{S}\right)$ is a finite union of primes. Thus, $Z_{R}\left(M^{\prime}\right)$ is a finite union of primes by Lemma 2. 
COROLlaRY 5. Let $R$ be a Z.D. ring. Then finitely generated $R$ modules are Z.D.

Proof. We proceed by induction on the number of generators. $n=1$ is the hypothesis. Let $M=\left\{m_{1}, \ldots, m_{n}\right\}, M^{\prime}=\left\{m_{1}, \ldots, m_{n-1}\right\}$ and $M^{\prime \prime}=M / M^{\prime}$. Then $M^{\prime}$ is Z.D. by inductive hypothesis. $M^{\prime \prime}$ is Z.D. since it is cyclic. Hence, $M$ is Z.D. by Proposition 4.

COROLlARY 6. Let $R$ be a ring with a finitely generated faithful Z.D. module M. Then $R$ is a Z.D. ring.

Proof. Say $M=\left\{m_{1}, \ldots, m_{n}\right\}$. Then the cyclic modules $\left\{m_{i}\right\}$ are Z.D. by Proposition 4. Hence, so is their direct sum, $\left\{m_{1}\right\} \oplus \cdots \oplus\left\{m_{n}\right\}=M^{\prime}$. The map $f: R \rightarrow M^{\prime}$ given by $f(r)=r m_{1}+\cdots+r m_{n}$ is a monomorphism since $M$ is faithful. But $f(R)$ is a Z.D. module by Proposition 4 and $f(R)$ is isomorphic to $R$. Thus, $R$ is a Z.D. $R$ module. That is, $R$ is a Z.D. ring.

Definitions. Let $R$ be a ring and $I$ an ideal of $R$. $I$ is primary if $a b \in I$ and $a \notin I$ implies $b^{n} \in I$ for some $n$. A ring is called Laskerian if every ideal is the intersection of a finite number of primary ideals.

Information on primary ideals, primary decomposition, and Laskerian rings abounds. See, for example, [1, Chapitre 4].

The next proposition is certainly known although $I$ have been unable to find a suitable reference for it since treatments of primary decomposition often assume $R$ Noetherian.

Proposition 7. Laskerian rings are Z.D.

Proof. Let $I$ be an ideal of $R$. Then $I=Q_{1} \cap \cdots \cap Q_{i}$ with $Q_{i}$ primary and no $Q_{i}$ containing the intersection of the others. Let $P_{i}$ be the radical of $Q_{i}$. Then $P_{i}$ is prime. I claim $Z(R / I)=P_{1} \cup \cdots \cup P_{n}$. Suppose $a b \in I$ and $b \notin I$. Then $b \notin Q_{i}$ for some $i$. Then $a b \in Q_{i}$ and $b \notin Q_{i}$ implies $b \in P_{i}$. Thus $Z(R / I) \subset P_{1} \cup \cdots \cup P_{n}$.

Pick $a \in P_{i}$ and $b \in Q_{1} \cap \cdots \cap \hat{Q}_{i} \cap \cdots \cap Q_{n}-I$ where ${ }^{\wedge}$ indicates omission. Then $a^{m} \in Q_{i}$ for some $m$. Hence $a^{m} b \in I$. Hence, there exists an $s \geqq 0$ such that $a^{s} b \notin I$ and $a\left(a^{s} b\right) \in I$. Thus, $a \in Z(R / I)$ and $P_{1} \cup \cdots \cup P_{n} \subset Z(R / I)$. Hence $Z(R / I)=P_{1} \cup \cdots \cup P_{n}$ as claimed.

The converse is false, for Bourbaki [1, p. 170, Exercise 19] shows that a rank 2 valuation ring is not Laskerian. Any valuation ring is Z.D. since in it any union of primes is a prime.

Definitions. Let $R$ be a ring and $M$ an $R$ module. Then Ass $(M)$ is the set of primes of $R$ which are the annihilators of elements"of $M$. If $\left\{m_{i}\right\}_{i \in I}$ is a set of elements of $M$, then $\left\{m_{i}\right\}_{i \in I}$ are weakly independent if $m_{i}$ is not in the submodule of $M$ generated by $\left\{m_{\}_{j \in I-\{i\}}}\right.$ for all $i \in I$. The $\left\{m_{i}\right\}_{i \in I}$ are strongly independent if no $m_{i}$ is 0 and for all $i \in I, r m_{i}$ in the submodule generated by $\left\{m_{j}\right\}_{j \in I-\{i\}}$ implies $r m_{i}=0$.

Proposition 8. Let $R$ be a ring and $M$ an $R$ module. If every weakly independent set of $M$ is finite, then any set of primes in Ass $(M)$ which are pairwise incomparable is finite. 
Proof. Let $\left\{P_{i}\right\}_{i \in I}$ be an infinite set of pairwise incomparable elements of Ass $(M)$. Say $P_{i}=$ ann $\left(m_{i}\right)$. Then $\left\{m_{i}\right\}_{i \in I}$ is not weakly independent. Thus for some $j, j_{i}, \ldots, j_{n} \in I$ and $r_{j_{k}} \in R, m_{j}=\sum_{k=1}^{n} r_{j_{k}} m_{j_{k}}$. Hence $P_{j} \supset \bigcap_{k=1}^{n} P_{j_{k}} . P_{j}$ prime implies $P_{j} \supset P_{j_{k}}$ for some $k$. This contradicts the choice of the $P$ 's.

THEOREM 9. Let $R$. be a ring and $X$ and $Y$ indeterminants over $R$. Then

(1) If $R$ is not Noetherian, $R[X]$ has an infinite weakly independent set.

(2) If $R$ has an infinite weakly independent set, then $R / I$ has an infinite strongly independent set for some ideal I of $R$.

(3) If $R$ has an infinite strongly independent set, then $R[Y]$ is not Z.D.

Proof. (1) Let $\left(a_{1}\right) \subset\left(a_{1}, a_{2}\right) \subset \ldots$ be a strictly increasing set of ideals of $R$. Then $\left\{a_{i} X^{i}\right\}_{i \in Z^{+}}$is an infinite weakly independent set. Suppose not. Then

$$
a_{j} X^{j}=\sum_{k=1}^{j-1} r_{k} a_{k} X^{k}+\sum_{k=j+1}^{n} r_{k} a_{k} X^{k}
$$

for some $n$ and some $r_{k} \in R[X]$. Comparing coefficients of $X^{k}$ and noting that only $\sum_{k=1}^{j-1} r_{k} a_{k} X^{k}$ has terms in $X^{j}$ on the right-hand side, we see that $a_{j} \in\left(a_{1}, \ldots, a_{j-1}\right)$. This is a contradiction.

(2) Let $\left\{r_{j}\right\}_{j \in J}$ be an infinite weakly independent set. Let $I$ be the ideal generated by $\left\{s_{j} r_{j} \mid s_{j} r_{j}\right.$ is in the ideal generated by $r_{k}$ where $\left.k \in J-\{j\}\right\}$ where it is understood that $r_{j}$ runs over all the elements of $\left\{r_{j}\right\}_{j \in J}$. Then if $r_{j}^{\prime}$ is the image of $r_{j}$ in $R / I$, $\left\{r_{j}^{\prime}\right\}_{j \in J}$ is an infinite strongly independent set of $R / I$. Suppose $t^{\prime} r_{j}^{\prime}$ is in the ideal generated by $\left(r_{k}^{\prime}\right)_{k \in J-\{j\}}, I_{j}^{\prime}$. Then $t r_{j}-m \in I$ where $m \in I_{j}$, the ideal generated by $\left(r_{k}\right)_{k \in J-\{j\}}$. Hence

$$
t r_{j}-m=\sum_{q=1}^{n} s_{j_{q}} r_{j}+\sum_{q=1}^{p} s_{k_{q}} r_{k_{q}}
$$

where $r_{k_{q}} \neq r_{j}$ for all $q$. Rearranging we get

$$
\left(t+\sum_{q=1}^{n} s_{j_{q}}\right) r_{j}=m+\sum_{q=1}^{p} s_{k_{q}} r_{k_{q}}
$$

is in $I_{j} . \sum_{q=1}^{n} s_{j_{q}} r_{j} \in I$ since $s_{j_{q}} r_{j}$ were generators of $I$. Hence, $t r_{j} \in I$ and $t^{\prime} r_{j}^{\prime}=0$ as desired.

(3) Let $\left\{r_{j}\right\}_{j \in J}$ be a countable strongly independent set of $R$. Let $a_{j}=$ ann $\left(r_{j}\right)$ and $\mathfrak{m}_{j}$ be a maximal ideal containing $a_{j}$. Several $\mathfrak{m}_{j}$ may be equal. (If they could be selected distinct, $R$ itself would fail to be Z.D.) $\left\{r_{j}\right\}$ is strongly independent when thought of in $R[Y]$. In $R[Y]$ we can expand the $\mathfrak{m}_{j}$ to countably many distinct maximal ideals $M_{j}^{\prime}$ of $R[Y]$ such that $M_{j}^{\prime} \cap R=\mathfrak{m}_{j}$. Hence, we can pick $M_{j}$ maximal in $R[Y]$ with $M_{j} \cap R=\mathfrak{m}_{j}$ and all the $M_{j}$ distinct. Let $I$ be the ideal generated by $\left\{r_{j} M_{j}\right\}_{j \in J}$. We remark $M_{j} \supset I$ for all $j$. For $M_{j} \supset\left(r_{j} M_{j}\right)$. If $j \neq k$, then $r_{j} r_{k}=r_{k} r_{j}$. Hence, $r_{k}$ annihilates $r_{j}$ since the $r_{i}$ are strongly independent. Thus, $M_{j} \supset$ ann $\left(r_{j}\right) \supset\left(r_{k}\right)$ and $M_{j} \supset\left(r_{k} M_{k}\right)$ for $j \neq k$. Let $r_{j}^{\prime}$ be the image of $r_{j}$ in $R[Y] / I$. 
If $r_{j}^{\prime} \neq 0$, then $\operatorname{ann}_{R[Y] / I}\left(r_{j}^{\prime}\right) \supset M_{j} / I$ and hence equals it since $M_{j} / I$ is maximal. Then, $Z(R[Y] / I) \supset \cup M_{j} / I$ which is not even contained in any finite union of primes. Thus, $R[Y]$ is not Z.D.

Suppose $r_{j}^{\prime}=0$. Then $r_{j}=\sum_{i=1}^{n} s_{i} r_{j_{i}} m_{j}+r_{j} m_{j}$ where $m_{j} \in M_{j}, m_{j_{i}} \in M_{j_{i}}$, and $r_{j_{i}} \neq r_{j}$ for all $i$. Then $\left(1-m_{j}\right) r_{j}$ is in the ideal generated by $\left(r_{k}\right)_{k \in J-\{j\}}$. Hence $\left(1-m_{j}\right) r_{j}=0$ by strong independence. Hence, $1-m_{j} \in$ ann $\left(r_{j}\right) \subset M_{j}$. This is a contradiction. Thus, $R[Y]$ is not Z.D.

Corollary 10. If $R[X, Y]$ is Z.D., then $R$ is Noetherian.

Proof. Immediate from Theorem 9.

The Hilbert basis theorem, the Lasker-Noether decomposition theorem, and Proposition 7 combine to give the converse of Corollary 10. Hence, Noetherian rings are the biggest class of rings closed under polynomial adjunction which is Laskerian. I do not know if one indeterminant is enough for Corollary 10.

The following is used in Proposition 13.

Proposition 11. If $R$ is a non-Noetherian ring which contains an infinite field $F$ as a subring with the same unit, then $R[X]$ is not $Z . D$.

Proof. Let $\left(f_{i}\right), i \in Z^{+}$, be countably many distinct elements of $F-\{0\}$. Let $\left(a_{1}\right) \subset\left(a_{1}, a_{2}\right) \subset \cdots$ be a strictly increasing set of ideals of $R$. Let

$$
\mathfrak{a}_{n}=\left\{r \in R \mid r a_{n} \in\left(a_{1}, \ldots, a_{n-1}\right)\right\} .
$$

Then the $a_{n}$ are all proper ideals of $R$. The set $b_{1}=a_{1}\left(X-f_{1}\right), b_{2}=a_{2}\left(x-f_{1}\right)\left(X-f_{2}\right)$, $\ldots, b_{n}=a_{n}\left(X-f_{1}\right) \cdots\left(X-f_{n}\right), \ldots$ is a weakly independent set of $R[X]$. For if $b_{n}=\sum_{i=1}^{n-1} r_{i} b_{i}+\sum_{i=n+1}^{m} r_{i} b_{i}$, then factoring out by $\left(X-f_{n+1}\right)$ gives $a_{n} f_{n}^{*} \sum_{i=1}^{n-1} r_{n}^{\prime} a_{i} f_{i}^{*}$ where $r_{i}^{\prime} \in R$ and $f_{i}^{*}$ are nonzero elements of $F$ and hence units of $R$. Thus $a_{n} \in\left(a_{1}, \ldots, a_{n-1}\right)$ which is a contradiction.

Let $B_{n}=\left\{r \in R[X] \mid r b_{n} \in\left(\left(b_{i}\right)_{\left.i \in Z^{+}-{ }_{(n)}\right)}\right)\right\}$. Then $B_{n} \subset\left(a_{n},\left(X-f_{n+1}\right)\right)$. Hence, the $B_{i}$ can be expanded to distinct maximal ideals $M_{n}$ and if $I=\left(b_{n} M_{n}\right)_{n \in Z^{+}}$, then $\bigcup M_{n} \subset Z(R[X] / I)$. Hence $R[X]$ is not Z.D. To prove $B_{n} \subset\left(\mathfrak{a}_{n},\left(X-f_{n+1}\right)\right)$ we suppose that $r b_{n}=\sum_{i=1}^{n-1} s_{i} b_{i}+\sum_{i=n+1}^{m} s_{i} b_{i}$, then factoring out by $\left(X-f_{n+1}\right)$ gives $r^{\prime} a_{n} f_{n}^{*}=\sum_{i=1}^{n-1} s_{i}^{\prime} f_{i}^{*} a_{i}$ where $f_{n}^{*}$ is a nonzero element of $F$. Thus $r^{\prime} a_{n}$ $=f_{n}^{*-1}\left(\sum_{i=1}^{n-1} s_{i} f_{i}^{*} a_{i}\right)$. Hence, $r^{\prime} \in \mathfrak{a}_{n}$ and $r \in\left(a_{n},\left(X-f_{n+1}\right)\right)$.

Corollary 10 indicates the scarcity of non-Noetherian Z.D. rings. We have already mentioned that arbitrary valuation rings are Z.D. So are rings with only finitely many primes. Finite direct sums of Z.D. rings are Z.D. by Proposition 4. The following gives a nontrivial class of Z.D. rings.

Proposition 12. Let $R$ be a semilocal Noetherian domain of Krull dimension 3 and let $R^{\prime}$ be the integral closure of $R$ in its quotient field. Then $R^{\prime}$ is $Z$.D.

Proof. By [4, p. 118, (33.10)(2)], $R^{\prime}$ has only finitely many maximal ideals $\mathfrak{m}_{1}, \ldots, \mathfrak{m}_{n}$. Hence for each $i$, there is an $m_{i} \in \mathfrak{m}_{\mathfrak{i}}$ not in any other $\mathfrak{m}_{j}$. Then $R^{\prime \prime}$ 
$=R\left[m_{1}, \ldots, m_{n}\right]$ is still semilocal Noetherian, 3-dimensional with integral closure $R^{\prime}$. There is a one-to-one correspondence between the maximal ideals of $R^{\prime}$ and $R^{\prime \prime}$ given by $\mathfrak{m}_{i} \leftrightarrow \mathfrak{m}_{i} \cap R^{\prime \prime}$. We proceed by induction on $n$ after replacing $R$ by $R^{\prime \prime}$. Let $n=1$ and $I$ be an ideal of $R^{\prime}$. If $Z_{R^{\prime}}\left(R^{\prime} / I\right)$ contains $\mathfrak{m}_{1} \cap R_{1}$, then it equals $\mathfrak{m}_{1}$ since $\mathfrak{m}_{1}$ is the only prime of $R^{\prime}$ containing $\mathfrak{m}_{1} \cap R$. Hence, $Z_{R^{\prime}}\left(R^{\prime} / I\right)$ is a finite union of primes. If $Z_{R^{\prime}}\left(R^{\prime} / I\right) \ngtr \mathfrak{m}_{1} \cap R$, there exists $f \in \mathfrak{m}_{1} \cap R-Z\left(R^{\prime} / I\right)$. Let $S=\left\{1, f, f^{2}, \ldots\right\}$. Then $Z_{R_{S}^{\prime}}\left(\left(R^{\prime} \mid I\right)_{S}\right)=Z_{R^{\prime}}\left(R^{\prime} / I\right) R_{S}$ by Lemma 1 and $Z\left(R^{\prime} / I\right)$ is a finite union of primes if and only if $Z\left(\left(R^{\prime} / I\right)_{S}\right)$ is by Lemma 2 . But $R_{S}^{\prime}$ is the integral closure of $R_{S}$ which is a 2-dimensional Noetherian ring. Hence $R_{S}^{\prime}$ is Noetherian by $[4$, p. $120,(33.12)]$ and $Z_{R_{S}^{\prime}}\left(\left(R^{\prime} / I\right)_{S}\right)$ is a finite union of primes.

Assume the proposition is true for all $n \leqq m$. Let $R^{\prime}$ have $m$ maximal ideals. Let $I$ be an ideal of $R^{\prime}$. If $Z_{R^{\prime}}\left(R^{\prime} / I\right) \supset\left(\mathfrak{m}_{1} \cap R\right) \cup \cdots \cup\left(\mathfrak{m}_{m} \cap R\right)$, then $Z_{R^{\prime}}\left(R^{\prime} / I\right)$ $=\mathfrak{m}_{1} \cup \ldots \cup \mathfrak{m}_{m}$ which is a finite union of primes. Otherwise there exists an $f \in\left(\mathfrak{m}_{1} \cap R\right) \cup \cdots \cup\left(\mathfrak{m}_{m} \cap R\right)-Z_{R^{\prime}}\left(R^{\prime} \mid I\right)$. Let $S=\left\{1, f, f^{2}, \ldots\right\}$. As before it is enough to prove that $Z_{R_{S}^{\prime}}\left(\left(R^{\prime} / I\right)_{S}\right)$ is a finite union of primes. But $R_{S}^{\prime}$ is the integral closure of $R_{S}$ and $R_{S}^{\prime}$, and the maximal ideals of $R_{S}$ and $R_{S}^{\prime}$ are in one-to-one correspondence. But $R_{S}$ is either 2-dimensional which forces $R_{S}^{\prime}$ to be Noetherian or $R_{S}$ is 3-dimensional with strictly fewer maximal ideals. Hence, $Z_{R_{S}^{\prime}}\left(\left(R^{\prime} / I\right)_{S}\right)$ is a finite union of primes and $R^{\prime}$ is Z.D. as desired.

Nagata in [4, p. 207] gives an example of a 3-dimensional Noetherian local ring, $R$, whose integral closure, $R^{\prime}$, is not Noetherian. Proposition 12 asserts $R^{\prime}$ will be Z.D. $R^{\prime}$ is a domain whose prime structure is at least as complicated as $R^{\prime}$ 's. In particular $R^{\prime}$ has many infinite sets of pairwise incomparable primes which could make $R^{\prime}$ fail to be Z.D. if they were the zero divisors on some $R^{\prime} \mid I$.

Nagata's example contains an imperfect (and hence infinite) field. $R[X]$ will be 4-dimensional with integral closure $R^{\prime}[X]$ which is not Z.D. by Proposition 11. Hence, Proposition 12 cannot be significantly strengthened. We state this as

Proposition 13. The integral closure of a 4-dimensional Noetherian domain can fail to be Z.D.

Lemma 14. Let $M$ be a Z.D. $R$ module and I a finitely generated ideal of zero divisors on $M$. Then $I$ is contained in the annihilator of some element $m \neq 0$ of $M$.

Proof. The proof is by induction on the number of generators of $I$. The case $n=1$ is obvious. Let $I=\left(i_{1}, \ldots, i_{n+1}\right)$. Expand $I$ to a prime ideal $P$ of zero divisors on $M$. Let

$$
N=\left\{m \in M \mid \exists s \geqq 1 \text { with } i_{n+1}^{s} m=0\right\} .
$$

Then $N$ is a submodule of $M$ and $i_{n+1} \notin Z(M / N) . Z(M) \subset Z(N) \cup Z(M / N)$. Both $N$ and $M / N$ are Z.D. by Proposition 4. Say $Z(N)=P_{1} \cup \cdots \cup P_{m}$ and $Z(M / N)=Q_{1} \cup \cdots \cup Q_{k} . P \subset Z(M)$ and $P \notin Q_{i}$ for any $i$. Hence, $P \subset P_{j}$ some $j$. Thus $I \subset Z(N)$. Then $I^{\prime}=\left(i_{1}, \ldots, i_{n}\right) \subset Z(N)$ and, hence, $I^{\prime}$ is contained in the 
annihilator of some $n^{\prime} \in N$. Thus there exists an $s \geqq 0$ such that $i_{n+1}^{s} n^{\prime} \neq 0$ and $i_{n+1}^{s+1} n^{\prime}=0$. Thus ann $\left(i_{n+1}^{s} n^{\prime}\right) \supset I^{\prime} \cup\left\{i_{n+1}\right\}$. Hence, ann $\left(i_{n+1}^{s} n^{\prime}\right) \supset I$ as desired.

Definition. Let $R$ be a ring and $A$ an $R$ module. The ordered sequence of elements $x_{1}, \ldots, x_{n}$ of $R$ is an $R$ sequence on $M$ if

(1) $\left(x_{1}, \ldots, x_{n}\right) A \neq A$, and

(2) for $i=1, \ldots, n, x_{i} \notin Z\left(A /\left(x_{1}, \ldots, x_{i-1}\right) A\right)$.

At this point we quote a theorem of Kaplansky on $R$ sequences which appears in [3, p. 86, Theorem 118].

TheOREM A. Let $x_{1}, \ldots, x_{n}$ be an $R$ sequence on $A$. Then the sequence obtained by interchanging $x_{i}$ and $x_{i-1}$ is an $R$ sequence on $A$ if and only if

$$
x_{i} \notin Z\left(A /\left(x_{1}, \ldots, x_{i-1}\right) A\right) .
$$

There are at least two proofs of the invariance of the length of a maximal $R$ sequence in the Noetherian case. The homological one breaks down for nondiscrete rank 1 valuation rings mod any nontrivial ideal. The second proof taken from [2] or [3, p. 88] adapts almost without change to the Z.D. case. In the Noetherian case, $R$ sequences are finite. I do not know this for Z.D. rings. Hence the following theorem is to be interpreted as allowing infinite $R$ sequences if they exist.

THEOREM 15. Let $R$ be a ring. A a Z.D. $R$ module, and I an ideal of $R$ with $I A \neq A$. Then any two maximal $R$ sequences on $A$ contained in $I$ have the same length.

Proof. It evidently suffices to prove the following: If $x_{i}, y_{i} \in I$ and $x_{1}, \ldots, x_{n}$ is a maximal $R$ sequence on $A$ and $y_{1}, \ldots, y_{n}$ is an $R$ sequence on $A$, then $y_{1}, \ldots, y_{n}$ is maximal. We proceed by induction on $n$.

$n=1$. After a change of notation we have the following setup: $x$ and $y$ are in $I$, they are nonzero divisors on $A$, and $I$ consists of zero divisors on $A / x A$. We must prove that $I$ consists of zero divisors on $A / y A$. Pick any $i \in I$. Then $(i, y)$ is a finitely generated ideal of zero divisors on $A / x A$. Hence, by Lemma 14 there is an element $u^{+} \neq 0$ in $A / x A$ annihilated by $(i, y)$. Restating this in $A$, we have $u \in A-x A$ with $(i, y) u \subset x A$. Say $y u=x v$ and $i u=x w$. Then we claim $v \notin y A$ and $i v \in y A$. Hence $i \in Z(A / y A)$ and $i \subset Z(A / y A)$ as desired. If $v=y a$, then $x v=x y a=y u$. Cancelling the $y$, since it is a nonzero divisor, yields $x a=u$. But $u \notin x A$. Next we show $i v \in y A$. For $x i v=i y u=y x w$. Cancelling the $x$ gives $i v=y w$.

General $n$. For brevity let us write

$$
B_{i}=A /\left(x_{1}, \ldots, x_{i-1}\right) A \text { and } C_{i}=A /\left(y_{1}, \ldots, y_{i-1}\right) A \text { for } i=1, \ldots, n \text {. }
$$

$C_{i}$ and $B_{i}$ are Z.D. for all $i$ by Proposition 4. Hence $\bigcup_{i=1}^{n}\left(Z\left(B_{i}\right) \cup Z\left(C_{i}\right)\right)$ is a finite union of primes. The existence of the $x_{i}$ and $y_{i}$ show that $I \notin Z\left(B_{i}\right)$ and $I \notin Z\left(C_{i}\right)$. Hence $I \notin \bigcup_{i=1}^{n}\left(Z\left(B_{i}\right) \cup Z\left(C_{i}\right)\right)$. Pick $z \in I-\bigcup_{i=1}^{n}\left(Z\left(B_{i}\right) \cup Z\left(C_{i}\right)\right)$. $z \notin Z\left(B_{n}\right)$ while $x_{n}$ is a maximal $R$ sequence on $B_{n}$. Now the fact that $z$ is not a member of any of $Z\left(B_{n-2}\right), Z\left(B_{n-3}\right), \ldots, Z\left(B_{1}\right)$, together with Theorem A, allows us to push $z$ ahead of the $x$ 's one step at a time until we reach the conclusion that 
$z, x_{1}, \ldots, x_{n-1}$ is an $R$ sequence on $A$. It is clearly maximal. Similarly $z, y_{1}, \ldots, y_{n-1}$ is an $R$ sequence on $A$. Now we pass to $A / z A$ on which we have two $R$ sequences of length $n-1: x_{1}, \ldots, x_{n-1}$ and $y_{1}, \ldots, y_{n-1}$, of which we know the first to be maximal. By induction $y_{1}, \ldots, y_{n-1}$ is a maximal $R$ sequence on $A / z A$, which implies that $y_{1}, \ldots, y_{n-1}, z$ is a maximal $R$ sequence on $A$. This implies that $y_{1}, \ldots, y_{n}$ is a maximal $R$ sequence on $A$ as desired.

I do not know if $R$ sequences can be permuted under radical assumptions as in the Noetherian case. However, using Theorem A and Proposition 14 we can replace any $R$ sequence by another which can be permuted.

\section{BIBLIOGRAPHY}

1. N. Bourbaki, Algèbre commutative, Chapitres 3, 4, Actualités Sci. Indust., no. 1293, Hermann, Paris, 1961. MR 30 \#2027.

2. I. Kaplansky, Commutative rings, University of Chicago, Chicago, Ill., 1968. (Mimeographed notes.)

3. - Commutative rings, Allyn and Bacon, Boston, Mass., 1970.

4. M. Nagata, Local rings, Interscience Tracts in Pure and Appl. Math., no. 13, Interscience, New York, 1962. MR 27 \#5790.

UNiVERSity of CALIFornia,

Los Angeles, California 90024 